\title{
Simulation and disturbance estimation of speed-controlled mechatronic drive systems
}

\author{
Chris Schöberlein ${ }^{1, *}$, Manuel Norberger ${ }^{1}$, Holger Schlegel ${ }^{1}$, and Matthias Putz ${ }^{1,2}$ \\ ${ }^{1}$ Institute of Machine Tools and Production Processes, Chemnitz University of Technology, Reichenhainer Straße 70, 09126 Chemnitz, \\ Germany \\ ${ }^{2}$ Fraunhofer-Institute for Machine Tools and Forming Technologies, Reichenhainer Straße 88, 09126 Chemnitz, Germany
}

\begin{abstract}
In the field of modern production systems, the process-parallel measurement of time-varying process forces and torques for high-level process monitoring is becoming increasingly important. Commonly utilized methods are based on additional sensors placed close to the working area. Unfortunately, this often leads to a higher complexity and additional costs due to the necessity of external hardware. An alternative approach is to evaluate the already available machine-internal signals of the subordinate drive systems. The process forces act on the load side of the machine axes in the form of disturbance forces and influence the drive-internal signals such as motor current or motor speed. To extract these disturbances, additional superimposed forces (e.g. friction and acceleration forces) and the influence of the feedback control as well as the mechanical system must be considered. Therefore, in the present paper, the application of various observer-based approaches for the estimation of load side disturbances on speedcontrolled mechatronic drive systems will be examined. The investigations are performed on a simulation model of a speed-controlled machine axis. After an introduction, three different disturbance observers will be presented. Subsequently, the paper describes the structure of the simulation model including its parameterization based on test rig measurements. Afterwards, the performance of the disturbance observers will be investigated and compared taking selected influencing factors like changing controller and inertia parameters into account. The paper closes with a summary and an outlook.
\end{abstract}

\section{Introduction}

In the field of production engineering, the prevailing process forces are a sensitive parameter for the indirect evaluation of the process state and its stability [1]. Usually, their measurement is realized by installing nearprocess sensors, for example in the form of piezo-based force measuring sensors or by means of capacitive or inductive displacement sensors [2, 3]. These sensors have advantages in terms of accuracy, dynamics and sensitivity due to the proximity to the working area. However, the process conditions (chips, temperature, cooling lubricant) may have negative effects on the lifespan of the sensors. In addition, an increased complexity of the overall system due to additionally required evaluation units and high requirements with regard to sensor integration have to be considered.

On the other hand, there are various approaches which are based on the already available control-internal signals and do not require any manipulation of the existing machine structure. However, one major obstacle is the reduced accuracy of this concepts which is highly dependent on the mechanical system and its state of motion. In addition, changes in friction torque and moment of inertia are affecting the quality of the estimation. Eventually, the quality of the measured signals is affected by the internal system clock of the machine control or the drive system, respectively.

In recent years, a variety of methods for the drivebased estimation of process forces has been developed. Common concepts were based on the evaluation of the motor currents [4-7] or the use of different kinds of state observers $[8,9]$ and were investigated for various motor types and mechanical systems. An alternative is provided in the form of model-based approaches based on disturbance observers, which consist of an order-reduced model of the mechanical part of the drive system. In this paper three disturbance observers are examined including a performance evaluation under variation of different influencing factors.

The paper has the following structure: in chapter 2 several disturbance observers will be presented. In addition to the conventional disturbance observer (DOB), the load side disturbance observer (LDOB) and the multi-encoder based disturbance observer (MEDOB) will be introduced. In chapter 3 , the modeling of the drive system based on a speed-controlled rotary drive test rig in MATLAB ${ }^{\circledR}$ Simulink ${ }^{\circledR}$ will be carried out. In particular, the model consists of the mechanical subsystem including effective friction torques and different model orders as well as the control loops for speed and current. Chapter 4 compares the observers by

Corresponding author: chris.schoeberlein@mb.tu-chemnitz.de 
simulating different disturbance effects and influencing factors. In former research, especially the effects of the drive controller settings or deviations in the parameters of the mechanical system were not examined in detail. Therefore, several settings of the speed controller as well as the robustness of the observers under variation of the load side inertia and the elastic coupling between input and output will be investigated.

\section{Types of Disturbance Observers}

In the following section, three common concepts of disturbance observers are examined in detail. With regard to the mechanical system, a two-mass system with one elastically coupled load inertia is assumed (figure 1, top). This system consists of a motor side moment of inertia $J_{m}$ and a load side moment of inertia $J_{l}$. The elastic coupling is defined by the torsional rigidity $c$ and the mechanical damping constant $d$. Friction torques act on the motor side $\left(T_{f, m}\right)$ and the output side $\left(T_{f, l}\right)$, respectively. Relevant disturbance torques $T_{\text {dist }}$ usually act on the load side only.

\subsection{Disturbance observer (DOB)}

The elementary structure for estimating load torques on mechatronic drive systems is the conventional disturbance observer as described in $[10,11]$ (figure 1, left). The estimation of the load side disturbance torque is based on the simplified assumption of a rigid coupling between motor and load and thus a single-mass system. The equation of motion of such a system is shown in equation (1).

$$
J \frac{d \omega_{m}}{d t}+T_{l}=I_{m} K_{t}
$$

$J$ denotes the total moment of inertia, $\omega_{m}$ the motor angular speed, $T_{l}$ the load torque including disturbance and total friction torque, $I_{m}$ the motor current and $K_{t}$ the torque coefficient. Usually it is assumed that fluctuations of the moment of inertia and the torque coefficient are negligibly small and therefore the nominal values of both parameters are utilized. How changes in the load side moment of inertia influence the estimation accuracy is examined in chapter 4. Due to the assumption of a rigid coupling between the input and output side, the disturbance torque is calculated as shown in equation (2). Note that $\widehat{T}_{f, m}$ and $\widehat{T}_{f, I}$ denote the models for the friction torques and $J_{m, n}$ and $J_{l, n}$ determine the moments of inertia on motor and load side, respectively.

$$
\widehat{T}_{d i s t} \approx I_{m} K_{t, n}-\widehat{T}_{f, m}-\widehat{T}_{f, l}-\left(J_{m, n}+J_{l, n}\right) \frac{d \omega_{m}}{d t}
$$

In addition, it is possible to implement a low-pass filter with the cut-off-frequency $g_{\text {dist }}$ to filter signal noise which arises by the discrete-time differentiation of the speed signal. Due to the reduced model order, the DOB is suitable for disturbance estimation on linear direct drives. However, electromechanical axes are usually characterized by an elastic coupling between input and output sides. This results in a reduced bandwidth and estimation accuracy of the DOB.

\subsection{Load disturbance observer (LDOB)}

To negotiate the disadvantages of the DOB, an observer structure with focus on the load side is suggested in [12, 13]. In contrast to the DOB, the structure shown in the center of figure 1 is based on the assumption of an elastically coupled load inertia and therefore utilizes the nominal spring constant $c_{n}$ and the angular difference between the input and output angles $\varphi_{l}-\varphi_{m}$. In addition, the observer includes a model of the load side friction torque $\widehat{T}_{f, l}$ as well as a load side moment of inertia $J_{l, n}$. This results in the following estimation equation for the disturbance torque:

$$
\widehat{T}_{\text {dist }} \approx c_{n}\left(\varphi_{m}-\varphi_{l}\right)-\widehat{T}_{f, l}-J_{l, n} \frac{d \omega_{l}}{d t}
$$

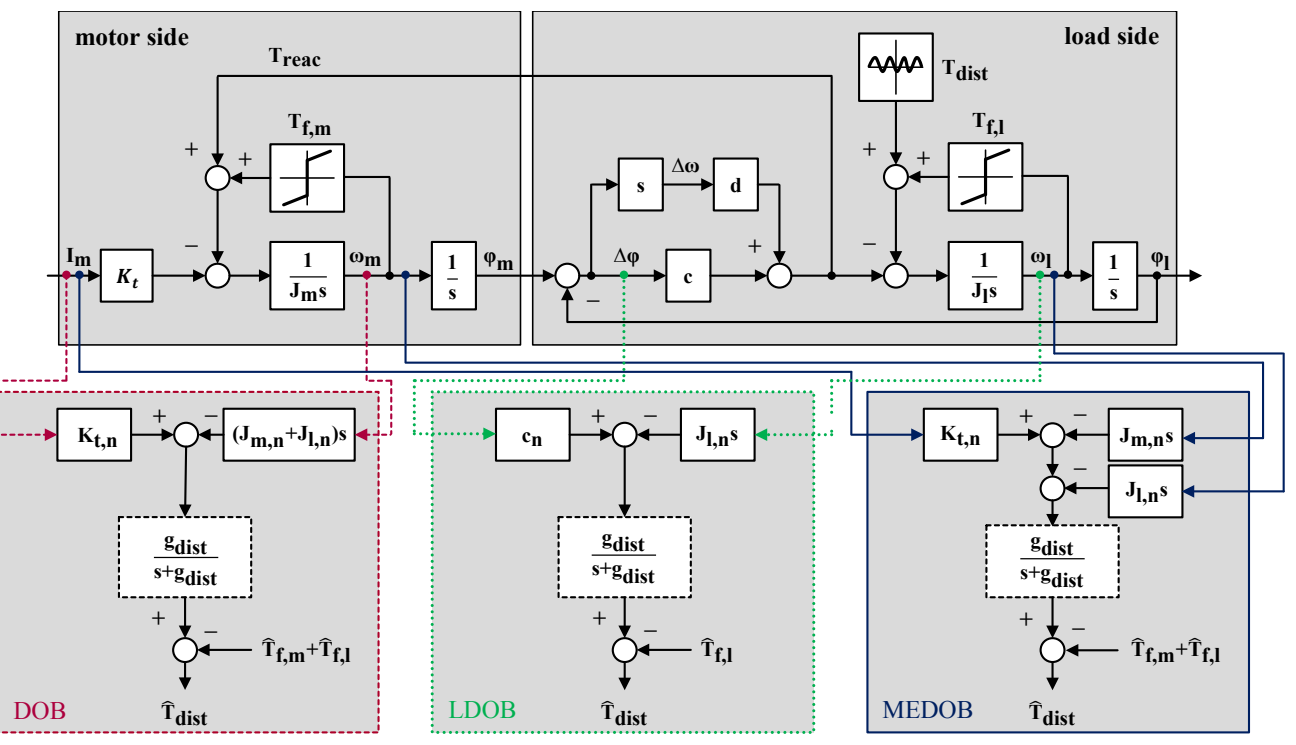

Fig. 1. Structure of the two-mass system including different disturbance observers and required input signals. 
One major obstacle for practical application of the LDOB is the required knowledge of the spring constant between motor and load side. In particular, the rigidy of linear feed axes (e.g. ball-screw drives) depends on the current axis position and typically has non-linear behavior [12]. Furthermore, a direct position measuring system to determine the load position is necessary. Additional difficulties arise in the practical identification of the friction torque on the load side, which requires the disassembly of the mechanical coupling.

\subsection{Multi-Encoder based disturbance observer (MEDOB)}

As a third option, the multi-encoder based disturbance observer (figure 1, right) is considered, as already presented in $[12,14]$. This approach is based again on the model concept of a two-mass system. The equations of motion of such a system are as follows:

$$
\begin{gathered}
J_{m} \frac{d \omega_{m}}{d t}=I_{m} K_{t}-T_{f, m}-T_{\text {reac }} \\
J_{l} \frac{d \omega_{m}}{d t}=T_{\text {reac }}-T_{f, l}-T_{\text {dist }}
\end{gathered}
$$

The reaction torque $T_{\text {reac }}$ is calculated as the torsional and damping forces transmitted via the elastic shaft:

$$
T_{\text {reac }}=c\left(\varphi_{m}-\varphi_{l}\right)+d\left(\omega_{m}-\omega_{l}\right)
$$

By combining equations (4), (5) and (6), the reaction torque is erased and a load side disturbance torque can be calculated according to the following estimation equation:

$$
\widehat{T}_{\text {dist }} \approx I_{m} K_{t, n}-J_{m, n} \frac{d \omega_{m}}{d t}-J_{l, n} \frac{d \omega_{l}}{d t}-\widehat{T}_{f, m}-\widehat{T}_{f, l}
$$

The application of this structure also requires a encoder on the load side, but does not depend on the knowledge of the coupling parameters such as spring or damping constants. Compared to the DOB, this leads to increased estimation dynamics with simultaneously higher accuracy. With regard to the experimental determination of the influence of friction, suitable results may already be achieved with a total friction model in relation to the motor or load speed.

\section{Simulation model}

The investigation of the observer-based methods for the estimation of disturbance torques on mechatronic drive systems is performed in MATLAB ${ }^{\circledR}$ Simulink $^{\circledR}$. Based on a corresponding test rig with known parameters (figure 2), the modeling of the mechanical subsystem including a simulation of the friction conditions will be carried out. Subsequently, the control loops for speed and current are presented. For this paper, the simulation was performed with a cycle time of $62.5 \mu \mathrm{s}$.

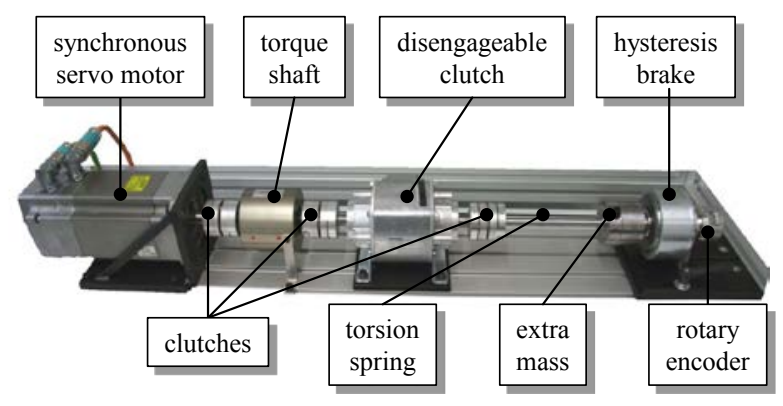

Fig. 2. Utilized drive system test rig.

\subsection{Modeling of mechanics and friction}

\subsubsection{Modeling of the mechanical system}

The transfer behavior of the mechanical subsystem of electromechanical axes is usually corresponding to a multi-mass mechanism. Depending on the mechanical complexity or the modeling quality, models of different complexity can be utilized to accurately reflect the real behavior.

A model widely used in control engineering for oscillating mechanics is the two-mass system, which was already mentioned in chapter 2 . Furthermore, an additional load inertia $J_{l, 2}$ can be coupled in the simulation. This leads to the model of a three-mass system. The elastic coupling of the additional load inertia is determined by the spring constant $c_{l 1, l 2}$ and the mechanical damping constant $d_{l l, l 2}$. Higher-order models are rarely used in industrial practice or research. Reasons are the high manual modeling effort and the only slight increase in accuracy. The parameterization of the models can be found in table 1 and is based on [15]. A comparison of the two model approaches in the frequency response is shown in figure 3 .

Table 1. Model parameters.

\begin{tabular}{|c|c|c|}
\hline & $\begin{array}{c}\text { Two-mass } \\
\text { system }\end{array}$ & $\begin{array}{c}\text { Three-mass } \\
\text { system }\end{array}$ \\
\hline$J_{m}\left(\mathrm{~kg} \cdot \mathrm{m}^{2}\right)$ & 0.000869 & 0.000869 \\
\hline$J_{l, l}\left(\mathrm{~kg} \cdot \mathrm{m}^{2}\right)$ & 0.000485 & 0.000485 \\
\hline$J_{l, 2}\left(\mathrm{~kg} \cdot \mathrm{m}^{2}\right)$ & - & 0.000685 \\
\hline$c_{m, l l}\left(\frac{\mathrm{Nm}}{\mathrm{rad}}\right)$ & 2150 & 2150 \\
\hline$c_{l l, l 2}\left(\frac{\mathrm{Nm}}{\mathrm{rad}}\right)$ & - & 1800 \\
\hline$d_{m, l l}\left(\frac{\mathrm{Nms}}{\mathrm{rad}}\right)$ & 0.026 & 0.026 \\
\hline$d_{l l, l 2}\left(\frac{\mathrm{Nms}}{\mathrm{rad}}\right)$ & - & 0.016 \\
\hline
\end{tabular}




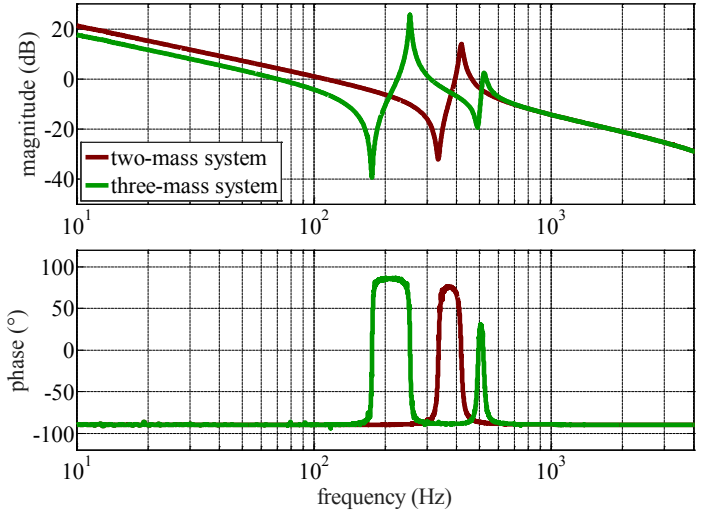

Fig. 3. Comparison of the models in the frequency response of the speed control plant.

\subsubsection{Modeling of the friction conditions}

In addition to the modeling of the transfer behavior of the mechanical system, the friction conditions at the test rig must be emulated. Therefore, experimental friction curves were recorded by repeated speed-controlled approaching of fixed speed points on the test rig. Subsequently, the average of these three curves was approximated utilizing a model approach as proposed in [16]. This approach represents the experimentally determined friction characteristic as a sum of four individual parts:

$$
T_{k}=T_{f, c}+T_{f, l i n}+T_{f, \text { exp }}+T_{f, \log }
$$

The first part consists of a constant, directiondependent friction component $T_{c}$ :

$$
T_{f, c}=\operatorname{sgn}(\omega) \cdot T_{c}
$$

A positive linear part takes speed-dependent friction effects into account and extends the model by a factor $\sigma$ to $T_{f, l i n}$ :

$$
T_{f, \text { lin }}=\sigma \cdot \omega
$$

The exponential friction torque component $T_{f, e x p}$ as shown in equation (11) includes the transition between static and sliding friction in the low-speed range. Therefore, the parameter $\omega_{\text {exp }, 0}$ defines the speed range in which the transition between static and sliding friction takes place. The form factor $\delta$ ultimately determines the exponential curve of the transition and can take positive and negative values for different friction surfaces. In addition, a static friction torque $T_{s}$ must be taken into account, which describes the maximum frictional force at standstill.

$$
T_{f, \exp }=\operatorname{sgn}(\omega) \cdot\left[\left(T_{s}-T_{c}\right) \cdot \exp \left(-\left|\frac{\omega}{\omega_{\exp , 0}}\right|^{\delta}\right)\right]
$$

The fourth friction component, which can be interpreted as rolling friction, is described as follows:

$$
T_{f, \log }=\operatorname{sgn}(\omega) \cdot\left[T_{\log } \cdot \ln \left(\frac{|\omega|}{\omega_{\log , 0}}+1\right)\right]
$$

Figure 4 shows the approximation of the measured friction conditions with the described model approach and its individual components.

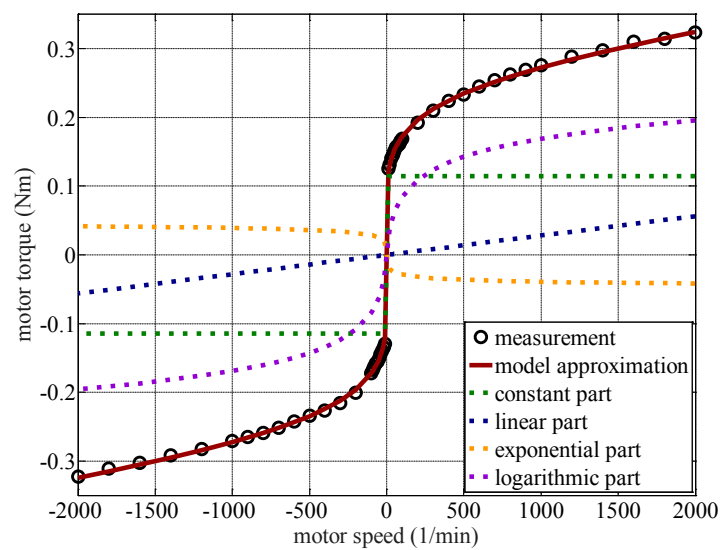

Fig. 4. Approximation of the experimental friction conditions and individual parts.

It can be stated that the approximation according to the proposed model approach simulates the friction conditions at the test rig very well and can therefore be utilized for the simulative investigations. Furthermore, by additional measurement of the motor side friction torque and its subsequent subtraction from the total friction torque, the friction influences on the load side for the two-mass system as well as the three-mass system can be mapped separately. This is of particular importance in the parameterization of the LDOB. The

\begin{tabular}{|c|c|c|c|c|c|c|c|}
\hline $\begin{array}{c}\text { friction } \\
\text { component }\end{array}$ & $T_{c}(\mathrm{Nm})$ & $\sigma\left(\frac{N m s}{r a d}\right)$ & $T_{s}(\mathrm{Nm})$ & $\omega_{\text {exp, },}\left(\frac{r a d}{s}\right)$ & $\delta$ & $T_{\text {log }}(\mathrm{Nm})$ & $\omega_{\text {log, } 0}\left(\frac{r a d}{s}\right)$ \\
\hline motor & 0.1158 & 0.00026 & 0.0664 & 0.6560 & -0.0098 & 0.0260 & 1.0900 \\
\hline load $_{1}$ & -0.0042 & 0.000049 & 0.0014 & 1.000 & -0.0062 & 0.0070 & 0.8813 \\
\hline $\operatorname{load}_{2}$ & -0.0042 & 0.000049 & 0.0014 & 1.000 & -0.0062 & 0.0070 & 0.8813 \\
\hline
\end{tabular}
parameterization of the friction approximation for the mechanical models used in the simulation is given in table 2.

Table 2. Friction model parameters. 


\subsection{Modeling of current and speed control}

\subsubsection{Supplementary model of the current control loop}

The feedback control of mechatronic drive systems is usually implemented in a cascaded form. For this paper, a speed control consisting of the two control loops for current and rotational speed is assumed. In particular, the determination of the influence of the speed controller on the estimation of disturbance torques is one main goal of the paper. For this reason, an established supplementary model was used for simulating the current loop, as it can be found in [17]. Based on experimental measurements on the test rig, a second order model approach with additional dead time has proved to be a suitable approximation. In figure 5, the frequency response of the closed current control loop of the test rig and the utilized supplementary model is shown.

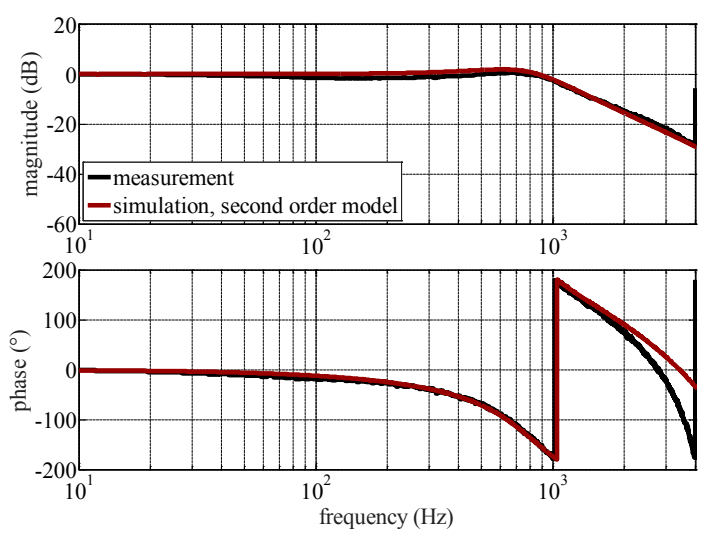

Fig. 5. Frequency response of the closed current loop and second-order model approximation.

As it can be seen, the model approach reflects the actual behaviour very well. Especially the course of the phase response is reproduced exactly over a wide frequency range. Furthermore, various additional current setpoint filters can be parameterized to attenuate possible resonance effects of the mechanical system.

\subsubsection{Modeling of the speed control loop}

In the course of this paper, the speed controller consists of a PI controller and the actual control values are provided by the motor encoder. Furthermore, it is possible to use an additional speed setpoint filter. Depending on the characteristics of the mechanical subsystem, different controller parameters (table 3 ) were examined, based on the investigations in [15]. The command and disturbance response in the frequency domain of all controller settings is shown in figure 6 . Note that the disturbance in the right picture is acting as disturbance torque on the load side of the mechanical system.

Setting No. 1 was deliberately chosen conservatively in order to minimize the influence of the speed controller on the estimation results. In the command frequency response, the setting is characterized by a limited bandwidth. This is also reflected in the disturbance behavior. Disturbances acting on the load side are barely adjusted by the speed control over a wide frequency range. Setting No. 2 is based on the established adjustment rule according to the symmetrical optimum, which is characterized by a high bandwidth without noticeable magnitude amplification. This setting requires the utilization of an additional speed setpoint filter to avoid high overshoots in the time domain. Furthermore, a current setpoint filter was installed to reduce resonance effects. Its parameterization is based on automatic selftuning procedures, which are commonly utilized by industrial distributed drive manufacturers. Concerning the disturbance behavior, setting No. 2 is capable of correcting disturbance influences over a relatively broad

Table 3. Speed Controller Settings.

\begin{tabular}{|c|c|c|c|c|c|c|c|}
\hline \multirow[b]{2}{*}{ No. } & \multicolumn{2}{|c|}{ Speed controller } & \multirow{2}{*}{$\begin{array}{c}\begin{array}{c}\text { Speed setpoint } \\
\text { filter (SSPF) }\end{array} \\
T_{S S P F}(s)\end{array}$} & \multicolumn{4}{|c|}{ Current setpoint filter (CSPF) } \\
\hline & $\begin{array}{c}K_{p} \\
\left(\frac{N m s}{r a d}\right)\end{array}$ & $T_{n}(s)$ & & $\omega_{F C N}(\mathrm{~Hz})$ & $D_{F C N}$ & $\omega_{F C D}(H z)$ & $D_{F C D}$ \\
\hline \multicolumn{8}{|c|}{ Two-mass system } \\
\hline 1 & 0.0802 & 0.00842 & - & - & - & - & - \\
\hline 2 & 1.3532 & 0.002 & 0.002 & 418.18 & 0.0 & 418.18 & 0.25 \\
\hline 3 & 2.0209 & 0.00552 & - & 418.18 & 0.2305 & 418.18 & 0.3982 \\
\hline \multicolumn{8}{|c|}{ Three-mass system } \\
\hline \multirow{2}{*}{4} & \multirow{2}{*}{1.6625} & \multirow{2}{*}{0.0025} & \multirow{2}{*}{0.0025} & 254.49 & 0.0 & 254.49 & 0.25 \\
\hline & & & & 520.18 & 0.0 & 520.18 & 0.25 \\
\hline
\end{tabular}



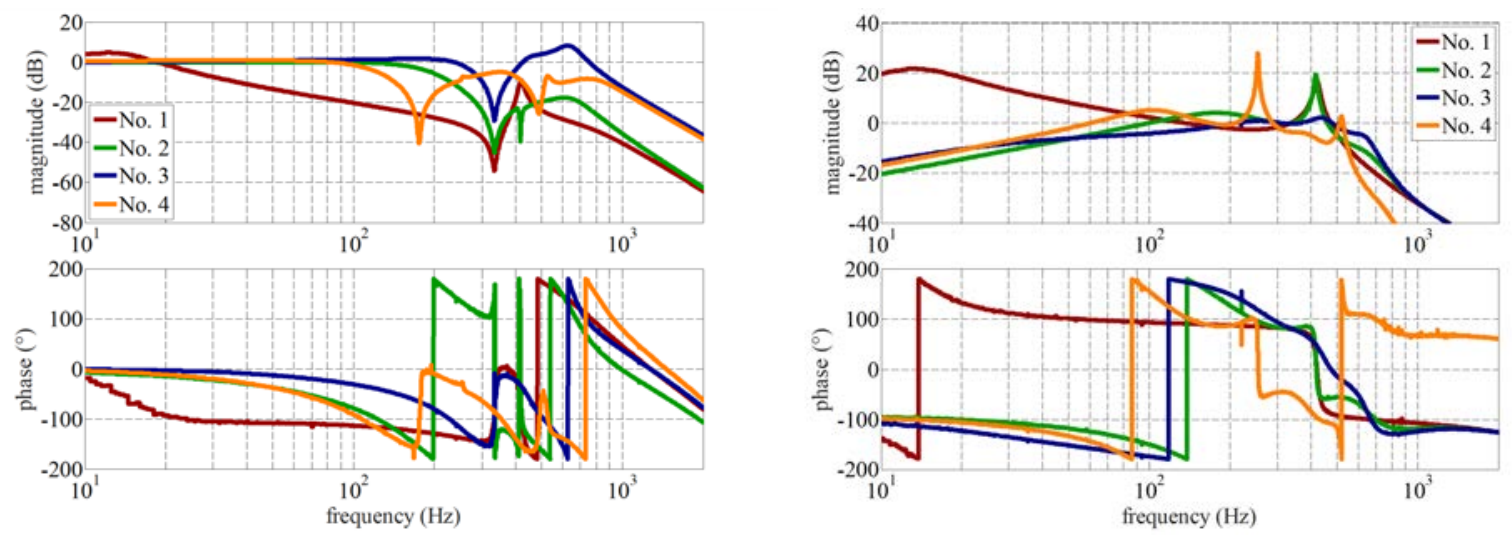

Fig. 6. Simulated command (left) and disturbance (right) frequency response for speed control settings No. 1-4.

frequency range up to approximately $100 \mathrm{~Hz}$. However, in the region of the mechanical natural frequencies the disturbances are amplified. Setting No. 3 was tuned utilizing the simulation-based optimization presented in [15]. This method includes optimized parameters for the current setpoint filters. Therefore, in the command frequency response a significantly higher control dynamic can be achieved. For the disturbance frequency response, the advantages of the optimized controller parameters lead to no significant amplification in the resonant frequency range.

In order to allow comparability with the two-mass system, the symmetrical optimum was also utilized for the three-mass system (No. 4). Overall, the bandwidth of the speed control compared to the two-mass system as a result of the changed coupling parameters is significantly reduced.

\section{Simulation results}

In the following section, the three disturbance observers presented in chapter 2 are implemented in the simulation model and examined with regard to their performance in the time and frequency domain. For better comparability, the actual motor torque $T_{m}$, which is typically available within the drive-internal control system, is also considered as a benchmark. In addition to the variation of the speed controller setting, the influences of parameter changes of the mechanical system are investigated. This includes changes in load inertia and spring constant as well as the extension of the mechanical system to a three-mass system. Note that the low-pass filters of the observers mentioned in chapter 2 were not implemented.

\subsection{Modification of the speed controller settings}

At the beginning, the influence of changing speed controller settings on the disturbance estimation of a two-mass system with the parameters listed in table 1 is examined. For this purpose, the speed controller settings No. 1-3 shown in table 3 are utilized. The disturbance torque on the load side was implemented in the form of a pseudorandom bit stream (PRBS) with a magnitude of $2 \mathrm{Nm}$. To avoid static friction effects, a rotational speed offset of $501 / \mathrm{min}$ was specified on the setpoint side.

Figure 7 shows the frequency responses of the motor torque and the disturbance observers, respectively. In the left picture it can be seen that the application of the DOB already has advantages over a pure evaluation of the motor torque. Although a more dynamic controller setting like No. 3 contribute to an increase in the
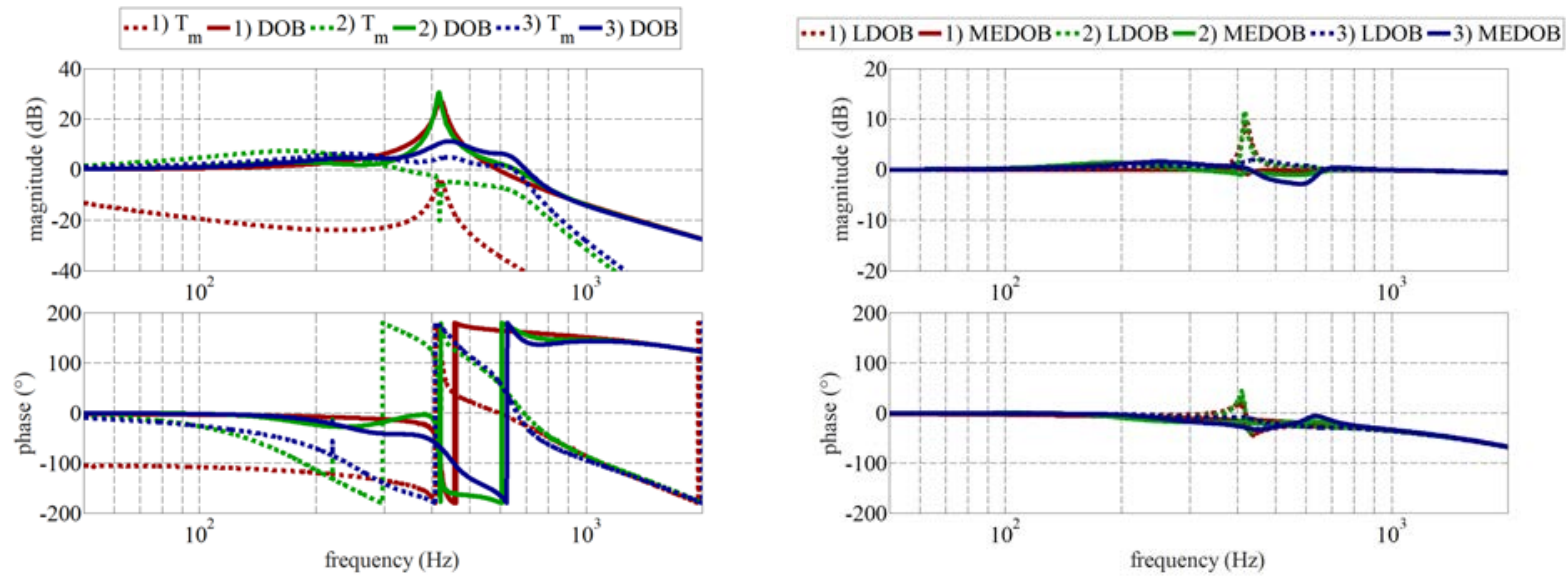

Fig. 7. Simulated frequency response for load side disturbance torque for speed controller settings No. 1-3. 
a) $\mathbf{T}_{\mathrm{m}}$

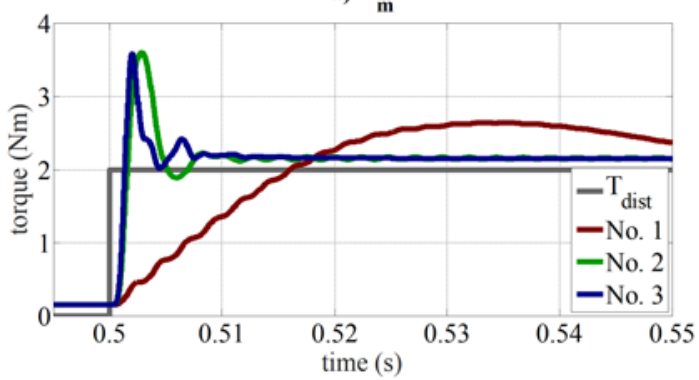

c) LDOB

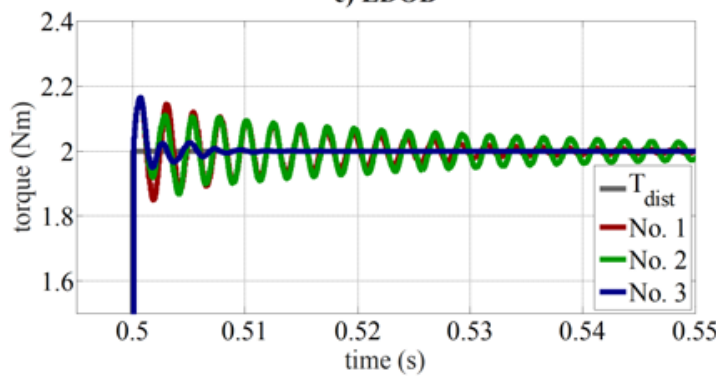

b) DOB

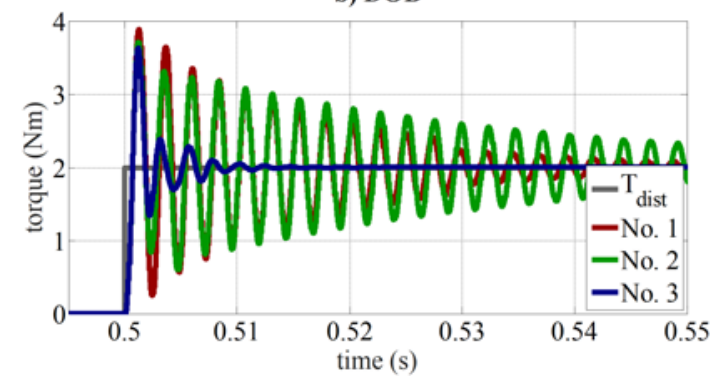

d) MEDOB

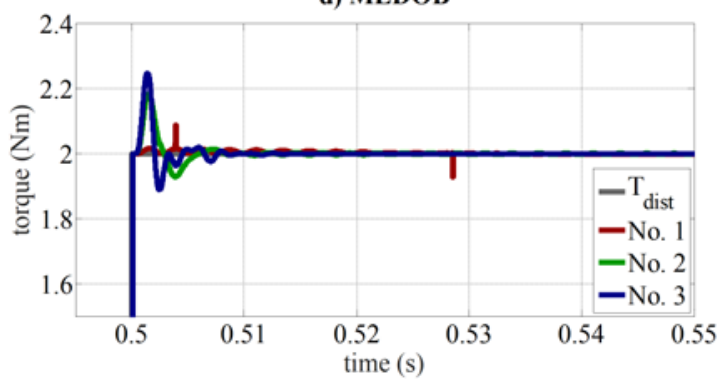

Fig. 8. Simulated step response for load-side disturbance torque for speed controller settings No. 1-3.

effective bandwidth of the motor torque, the existing deviations in the phase response are only partially compensated. In contrast, the DOB has no significant phase shift over a wider frequency range up to approximately $120 \mathrm{~Hz}$ for all controller settings. For the settings No. 1 and 2, the effects of the elastically coupled mechanics clearly appear in the magnitude characteristic. These effects can be partially compensated by the optimized speed controller settings (No. 3).

In the right picture, LDOB and MEDOB are compared. Note that due to the smaller deviations in the magnitude response its scaling was adjusted. Regardless of the controller parameterization, the estimation quality is enhanced for an even broader frequency range without significant phase shift for both observer types. In case of the LDOB, there is a noticeable increase in magnitude in the region of the natural frequency for runs No. 1 and 2. However, it is much lower compared to the DOB and can also be countered by an optimized controller setting as in run No. 3. For the MEDOB, the resonance point has no notable effects in the magnitude response even for controller settings No. 1 and 2. However, the more dynamic controller setting No.3 leads to slight deviations in the range of higher frequencies, which is also expressed in the closed loop command frequency response of this setting (see figure 6).

The results in the frequency domain can be confirmed by applying a step disturbance torque and a subsequent examination in time domain. In figure $8 \mathrm{a}-\mathrm{d}$, the simulation model is again operating with a speed offset of $501 / \mathrm{min}$. At a simulation time of $t_{\text {sim }}=0.5$ seconds, a step disturbance torque $T_{\text {dist }}$ with a magnitude of $2 \mathrm{Nm}$ was added on the load side.

The response of the motor torque in figure $8 \mathrm{a}$ shows significant differences depending on the controller setting. All three runs have in common that a constant deviation from the course of the disturbance torque due to unrecognized friction effects can be seen. In case of the DOB, the generally increased dynamics is also recognizable in the time domain. For the runs No. 1 and 2 , the excitation of the natural frequency is clearly visible. The best results are achievable utilizing the simulation-based optimized setting No. 3. For the LDOB and MEDOB equally a significantly reduced overshoot and better damping of the oscillations occurs. While the optimized controller setting No. 3 leads to an improved disturbance estimation for the LDOB, a high estimation accuracy for setting No. 1 can be determined for the MEDOB.

In summary, an optimized setting of the speed control has certain advantages, in particular when utilizing the motor torque or the conventional DOB. However, improvements in the range of mechanical resonances can be achieved for the LDOB by means of optimized speed control settings. The MEDOB profits in particular from conservative or established speed control setting rules (e.g. symmetrical optimum) and overall appears much more robust than the remaining strategies. Note that the deviations of the LDOB and MEDOB result from the differentiation of the actual speed values. This effect would increase for higher sample times of the control loops.

\subsection{Modification of load side moment of inertia}

As mentioned in chapter 2, the nominal values for the moment of inertia are used for the parameterization of the observers. In practical applications, especially the values on the load side are not constant, for example due to changing workpieces or modifications of the mechanical configuration. In the frequency response of the mechanical system, this variation manifests in a vertical shift of the magnitude response in the range of frequencies smaller than the natural frequency and a 


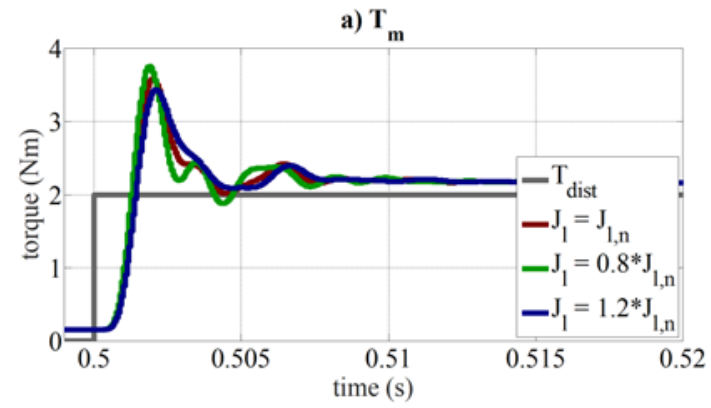

c) LDOB

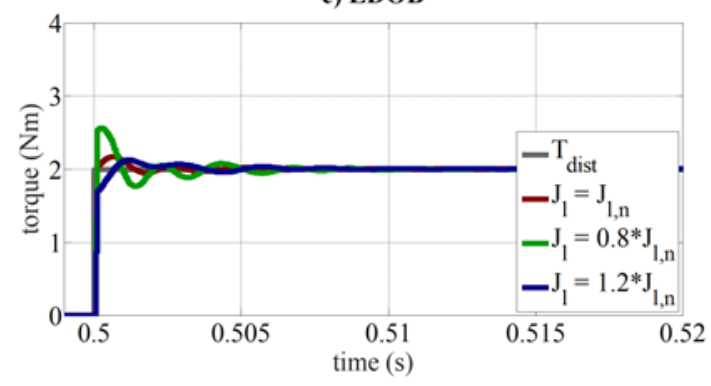

b) DOB

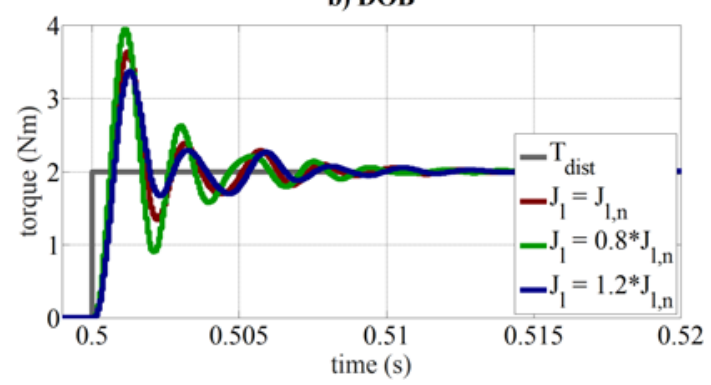

d) MEDOB

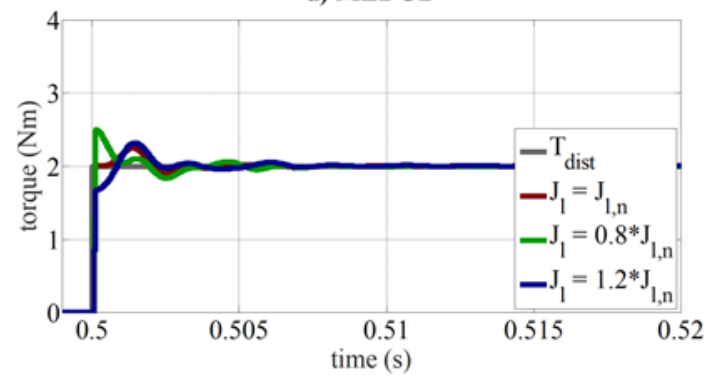

Fig. 9. Simulated step response for load-side disturbance torque with variation of load side inertia for speed controller settings No. 1-3.

horizontal displacement of the resonance points. Eventually, this has a direct impact on the quality of the drive control itself. Therefore, several methods for parameter identification [18] were developed in recent years which are able to detect these changes and adapt the controller parameters accordingly. However, these methods are currently rarely used in the industrial practice. For this reason, it is of particular interest how changes in the load side moment of inertia affect the estimation accuracy of the individual observers.

Figure 9 shows the step response of the observers as well as the motor torque with variation of the load side inertia to 80 or 120 percent of the nominal value. For all investigations, speed controller setting No. 3 was selected. To avoid disturbing static friction effects, again a speed offset of $501 / \mathrm{min}$ was specified.

For all the structures shown in figure 9 a-d, it can be stated that deviations in moment of inertia on the load side have only a minor influence on the estimation results. However, all methods have in common that a reduced load side moment of inertia (green curves) leads to higher overshoot and oscillations. This effect increases for higher deviations to the nominal values. In contrast, a higher moment of inertia (blue curves) leads to slightly reduced estimation dynamics, but smaller overshoots. Especially for the LDOB and MEDOB, there are only slight differences, which can be interpreted as improved robustness against variable load inertias. Note that larger deviations of the nominal inertia or a less robust setting of the speed controller parameters and current setpoint filters may have a negative impact on the estimation accuracy.

\subsection{Modification of the spring constant}

In addition to the process-related change of the load side inertia, it is possible that variations occur in the coupling parameters between motor and load side. For example, on linear feed axes (e.g. ball screw drive) changes in the load position or progressive wear of the mechanical elements (e.g. loss of pre-tension) affect the rigidity [19]. In the frequency response of the mechanical system, this variation manifest in a shift of the resonant and antiresonant frequency in the abscissa direction. Figure $10 \mathrm{a}-\mathrm{d}$ show the reaction of the motor torque and the observer structures again utilizing speed controller setting No. 3. The variation of the spring constant amounts 80 or 120 percent of the nominal value, respectively. As before, a step disturbance with a magnitude of $2 \mathrm{Nm}$ at the simulation time $t_{\text {sim }}=0.5$ seconds was applied on the load side. To neglect static friction effects, a speed offset of $501 / \mathrm{min}$ was predetermined.

For the course of the motor torque and DOB (Figure 10a and $\mathrm{b}$ ) the variation of the spring constant ultimately leads to a modified estimation dynamic. A more rigid elastic coupling (blue curves) leads to a faster response of the disturbance magnitude and simultaneous only slight changes in overshoot. For the LDOB, a change in the rigidity directly affects the estimation results. Thereby, an increased spring constant generates static lower estimations, while their reduction leads to too high approximations. In contrast, the variation of the spring constant has no real impact on the MEDOB, which can be justified by reference to equations (4), (5), (6) and (7). Smaller deviations between the curves again result from the differentiation of the rotational speed of motor and load side.

\subsection{Modification of the mechanical system}

In industrial practice, the mechanical subsystems are characterized by several elastically coupled masses of 


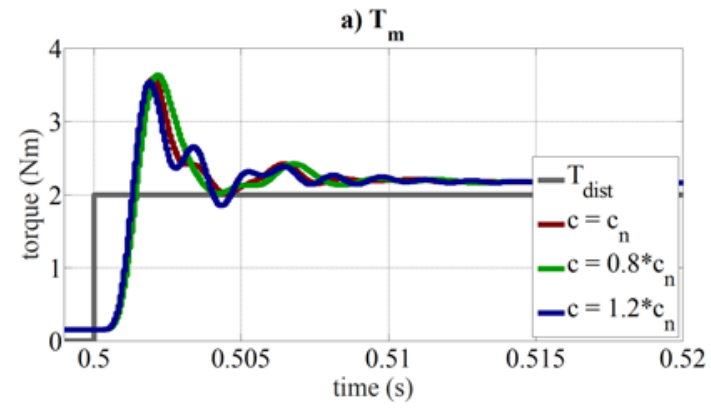

c) LDOB

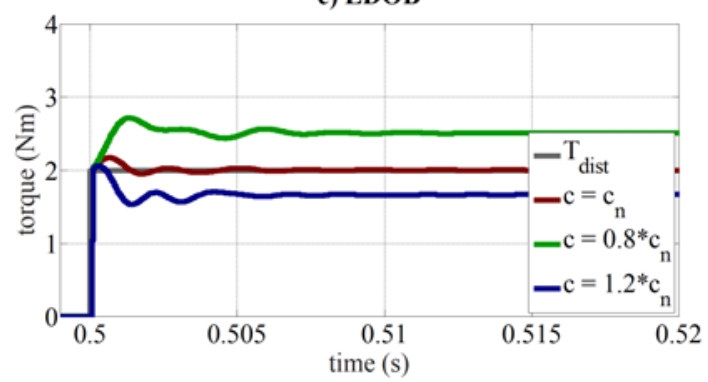

b) DOB

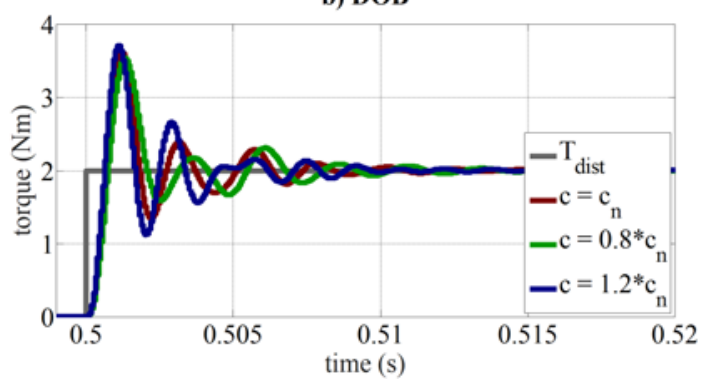

d) MEDOB

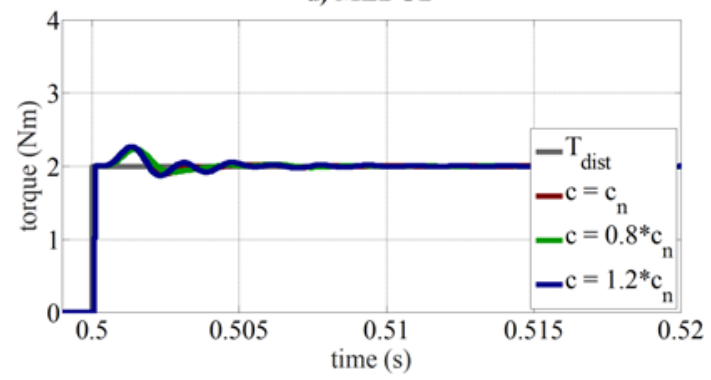

Fig. 10. Simulated step response for load-side disturbance torque with variation of the spring constant for speed controller settings No. 1-3.

inertia. Therefore, the simplification of the real system in the form of a two-mass is not always durable. In order to investigate the robustness of the observer structures more precisely, a second load inertia is implemented in the simulation model. The corresponding simulation parameters refer to table 1 and table 2, respectively. For the LDOB, the additional load side moment of inertia $J_{l, 2}$ was utilized as nominal value $J_{l, n}$. The nominal spring constant $c_{n}$ was calculated according to equation (13):

$$
\frac{1}{c_{n}}=\frac{1}{c_{m, l l}}+\frac{1}{c_{l 1, l 2}}
$$

For the MEDOB, it has proven to be beneficial to distribute the moment of inertia $J_{l, 1}$ in equal parts on the motor and load side. Therefore, $J_{m, n}$ and $J_{l, n}$ are calculated as follows:

$$
J_{m, n}=J_{m}+\frac{J_{l, 1}}{2}
$$

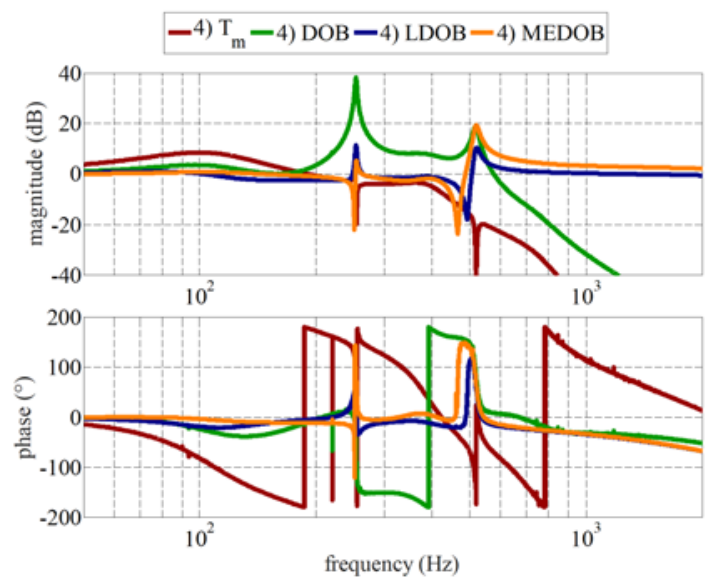

$$
J_{l, n}=J_{l, 2}+\frac{J_{l, 1}}{2}
$$

For the investigations in the time and frequency domain, the same disturbance parameters as for the twomass system are applied. Note that the parametrization of the speed controller and current setpoint filters is limited to setting No. 4 (table 3 ). The corresponding response behavior in the frequency and time domain is shown in figure 11.

As before, the DOB has only a slight phase shift compared to the motor torque over a wider frequency range (up to about $70 \mathrm{~Hz}$ ). However, the course of the magnitude is characterized by distinct peaks in the region of the mechanical resonance points. In comparison to the results of the two-mass system, slight deviations in magnitude are already recognizable for the DOB even in lower frequency ranges. In the time domain, the excitation of the mechanical natural

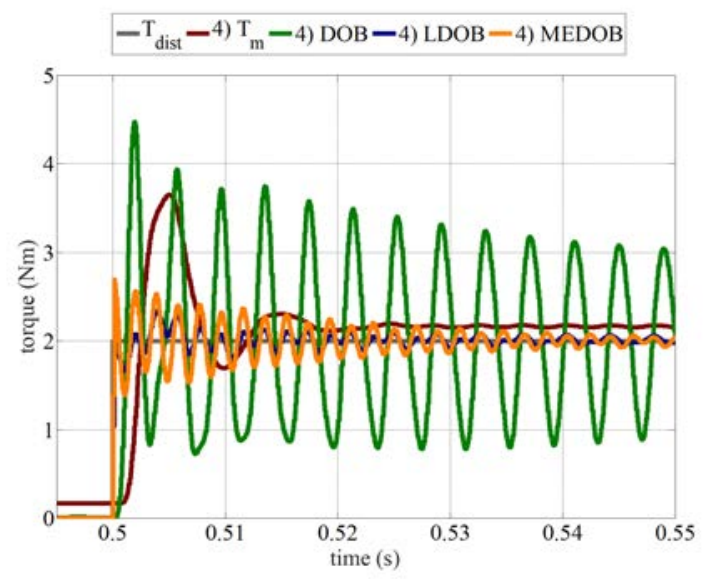

Fig. 11. Simulated response for load-side disturbance torque in frequency and time domain for speed controller setting No. 4. 
frequencies can be clearly recognized for a step disturbance, which leads to distinct and slightly attenuated oscillations. This could be prevented by a suitable filtering as mentioned in chapter 2. However, this may have a negative impact on the dynamics of the estimation.

The smallest deviations in magnitude are obtained by the LDOB, which, however, start in lower frequency ranges at about $100 \mathrm{~Hz}$. In the time domain, the oscillations are significantly less distinct compared to the DOB and decrease faster. When comparing the results with those of the two-mass system, the quality of the estimation is only slightly affected by the modified mechanical structure.

Again, the MEDOB achieves suitable estimation results compared to the other approaches. In the frequency domain, only slight deviations are detectable in the magnitude and phase response up to $200 \mathrm{~Hz}$. The excitation of the mechanical resonance frequency at $518 \mathrm{~Hz}$ is also recognizable in the time domain by significant oscillations in case of a step disturbance excitation. However, these oscillations decline much faster compared to the DOB. Again, it is possible to further improve the quality of the estimation by an appropriate filtering as mentioned in chapter 2 .

\section{Conclusion \& Outlook}

The application of model-based approaches in the form of disturbance observers is suitable for estimating load side disturbances on speed-controlled mechatronic drive systems. Depending on the complexity of the observer structure and the characteristics of the drive system, results of different quality are achievable. To investigate the individual structures, a simulation model was created and parameterized based on experimental measurements on a drive test rig. It enables the emulation of mechanical systems with variable model order and coupling parameters as well as different settings of the speed controller.

For disturbance estimation, a solitary evaluation of the motor torque has turned out as the simplest option, but it particularly depends on the setting of the speed control and is not able to take friction effects into account. In contrast, the conventional disturbance observer has clear advantages in terms of estimation dynamics. For elastically coupled mechanical systems, however, its application is not recommended. The LDOB achieves very good estimation results regardless of the mechanical subsystem and speed controller settings. However, it requires the exact knowledge of the spring constant as well as the friction torque of the load side, which is difficult to identify especially in industrial applications. An appropriate compromise is provided by the MEDOB, which obtains a high estimation accuracy, especially for two-mass systems. Furthermore, the quality of the estimation is barely affected by changes in the coupling parameters. Merely mechanical systems of higher order have a limiting effect, whereby undesired oscillations should be prevented with a suitable signal filtering.
Future investigations should focus on the enhancement of the simulation model accuracy including consequential effects on the observer structures. For example, the detailed modeling of the current control or the implementation of quantized encoder signals should be considered. In addition, the influence of appropriate signal filters to avoid unwanted oscillations caused by the elastic coupling of the inertia masses have to be investigated. For example, a separate filtering of the input and output signals of the observers offers further improvements. Another research goal is the application on position-controlled, linear feed axes. An experimental verification, for example on a machine tool, is also necessary.
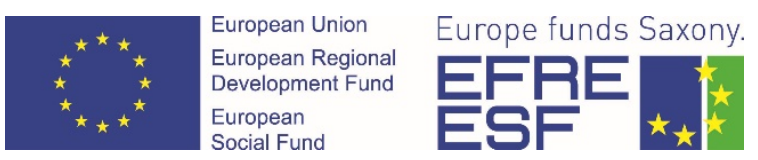

Funded by the European Union (European Social Fund) and the Free State of Saxony.

\section{References}

1. B. Bindu, B. Vinod. Measurement of Cutting Forces in CNC Turning Centers: A Review, International. Journal of Mechanical Engineering, 3(5): 77-87, (2015)

2. M. Rizal, J. Ghani, M. Nuawi, C. Haron. A Review of Sensor Systems and Application in Milling Processes for Tool Condition Monitoring, Research Journal of Applied Sciences, Engineering and Technology, 7(10): 2083-2097 (2014)

3. D. Dimla. Sensor Signals for Tool-Wear Monitoring in Metal Cutting Operations - a Review of Methods, International Journal of Machine Tools and Manufacturing, 40: 1073-1098 (2000)

4. J. Stein, K. Shin. Current Monitoring of Controlled DC Spindle Drives, Journal of Dynamic Systems, Measurement and Control, 108: 189-295 (1986)

5. Y. Altintas. Prediction of Cutting Forces and Tool Breakage in Milling from Feed Drive Current Measurements, Journal of Engineering for Industry, 114: 386- 392 (1992)

6. G. Kim, N. Chong. Indirect Cutting Force Measurement Considering Frictional Behaviour in a Machining Center using Feed Motor Current, International Journal of Advanced Manufacturing Technology, 15: 478-484 (1999)

7. R. Sato, M: Hasegawa, K. Shirase. Cutting Force Monitoring based on the Frequency Analysis of Feed Motor Torques, Journal of SME Japan (2013)

8. T. Kim, J. Woo, D. Shin, J. Kim. Indirect Cutting Force Measurement in multi-axis simultaneous NC Milling Processes, International Journal of Machine Tools and Manufacture, 39: 1717-1731 (1999)

9. G. Pritschow, J. Bretschneider, S. Fritz. Reconstruction of Process Forces within Digital 
Servodrive Systems, Annals of the German Academic Society for Production Engineering, 5(1): 73-78 (1999)

10. T. Murakami, F. Yu, K. Ohnishi. Torque Sensorless Control in Multidegree-of-Freedom Manipulator, IEEE Transactions on Industrial Electronics, 40(2): 259-265 (1993)

11. Y. Kakinuma, Y. Sudo, T. Aoyama. Detection of chatter vibration in end milling applying disturbance observer, CIRP Annals - Manufacturing Technology, 60: 109-112 (2011)

12. C. Mitsantisuk, M. Nandayapa, K. Ohnishi, S. Katsura. Design for Sensorless Force Control of Flexible Robot using Resonance Ratio Control based on Coefficient Diagram Method, Journal for Control, Measurement, Electronics, Computing and Communications, 54(1): 62-73 (2013)

13. S. Katsura, J. Suzuki, K. Ohnishi. Pushing Operation by flexible Manipulator Taking Environmental Information into Account, IEEE Transactions on Industrial Electronics, 53(5): 16881697 (2006)

14. Y. Yamada, Y. Kakinuma. Sensorless Cutting force estimation for full-closed controlled ball-screw- driven stage, International Journal of Advanced Manufacturing Technology, 87: 3337-3348 (2016)

15. K. Hipp. Einsatz hybrider Optimierungsverfahren zur Inbetriebnahme elektromechanischer Systeme, Berichte aus dem IWU, 98 (2017)

16. M. Kunc. Identifikation und Modellierung von nichtlinearen Dämpfungseffekten in Vorschubachsen für Werkzeugmaschinen, Ergebnisse aus der Produktionstechnik, 11 (2013)

17. S. Hofmann. Identifikation parametrischer Modelle für geregelte, elektromechanische Achsen mit modifizierter, sukzessiver Polkompensation, Berichte aus dem IWU, 69 (2012)

18. A. Hellmich, K. Hipp, H. Schlegel, R. Neugebauer. Parameter identification of NC-axes during regular operation of a machine tool, Advanced Materials Research, 1018: 419-426 (2014)

19. D. Maier. Sensorlose online Zustandserfassung von Vorschubantriebskomponenten in Werkzeugmaschinen, Berichte aus dem institute für Maschinenelemente, 157 (2015) 